\title{
Analytical solution of differential equation with cubic nonlinearity
}

\author{
Tatyana Inkhireeva \\ Applied Mathematics department \\ National Research Tomsk Polytechnic University \\ Tomsk, Russia \\ tai2@tpu.ru
}

\author{
Alexander Kozlovskikh \\ Applied Mathematics department \\ National Research Tomsk Polytechnic University \\ Tomsk, Russia \\ avk55@tomcity.net
}

\begin{abstract}
Cauchy problem solution for nonlinear differential equation. Source of solution error and way of eliminating it is studied. Solution obtained with suggested method is compared with solution obtained with builtin MATLAB functions.
\end{abstract}

Keywords-Cauchy problem, equilibrium points, Poincare secant, fundamental system of solutions.

\section{INTRODUCTION}

One of the questions in the orbit accelerators theory is studying behavior of charged particles in median plane of torus-shaped vacuum chamber. The behavior of the particles is studied both in stable and unstable oscillations region. Mathematically the question is reduced to the following equation

$$
\frac{d^{2} x}{d \theta^{2}}+x \cdot n(x)=0
$$

where $\mathrm{n}(\mathrm{x})$ is a differential characteristic of magnetic field decrease, which is created inside accelerator chamber. Function $\mathrm{n}(\mathrm{x})$ is obtained by magnetic field measurements processing. It is quite properly approximated by cubic polynomial. There is a nonlinear differential equation for one kind of orbit accelerators [1]. Solving the equation is a subject for study.

$$
\frac{d^{2} x}{d \theta^{2}}+0.298 \cdot x-0.0023 \cdot x^{3}=0
$$

Modify it into

$$
\frac{d^{2} x}{d \theta^{2}}+\omega(x)^{2} \cdot x=0
$$

where

$$
\omega(x)^{2}=\left(0.298-0.0023 \cdot x^{2}\right)
$$

The idea underlying the analytical solution is that having modified (1) into (2) and considered $\omega\left(x_{i}\left(\theta_{i}\right)\right)$ as const for small quantity ${ }_{\Delta} \theta$ ( $\theta_{i}$ is left boundary of interval $\theta_{i}+{ }_{\Delta} \theta$ ) we deduce homogeneous linear differential equation with constant coefficients. Solution of the equation is known:

$$
x=a \cdot \cos \left(\omega_{i} \cdot \theta\right)+b \cdot \sin \left(\omega_{i} \cdot \theta\right)
$$

Let us consider in greater detail Cauchy problem solution at the first step.

We are given initial conditions $\left(x_{0}, p x_{0}\right.$ is the derivative of $\left.x_{0}, \theta_{0}\right)$. Unknown coefficients can be found by differentiating general solution (3). Cauchy problem solution can be written in matrix representation.

$$
\begin{gathered}
\left(\begin{array}{l}
a_{0} \\
b_{0}
\end{array}\right)=\left(\begin{array}{cc}
\cos \left(\omega_{0} \cdot \theta_{0}\right) & \frac{-\sin \left(\omega_{0} \cdot \theta_{0}\right)}{\omega_{0}} \\
\sin \left(\omega_{0} \cdot \theta_{0}\right) & \frac{\cos \left(\omega_{0} \cdot \theta_{0}\right)}{\omega_{0}}
\end{array}\right) \cdot\left(\begin{array}{c}
x_{0} \\
p x_{0}
\end{array}\right) \\
\left(\begin{array}{c}
x\left(\theta_{0}+\Delta \theta\right) \\
p x\left(\theta_{0}+\Delta \theta\right)
\end{array}\right)=\left(\begin{array}{cc}
\cos \left(\omega_{0} \cdot\left(\theta_{0}+\Delta \theta\right)\right) & \sin \left(\omega_{0} \cdot\left(\theta_{0}+\Delta \theta\right)\right) \\
-\omega_{0} \cdot \sin \left(\omega_{0} \cdot\left(\theta_{0}+\Delta \theta\right)\right) & \omega_{0} \cdot \cos \left(\omega_{0} \cdot\left(\theta_{0}+\Delta \theta\right)\right)
\end{array}\right) \cdot\left(\begin{array}{l}
a_{0} \\
b_{0}
\end{array}\right)
\end{gathered}
$$

Coordinate and its derivative values in (4) are the initial values for the solution at the next step $\Delta \Theta$. In accordance with (2a) frequency is

$$
\omega\left(x\left(\theta_{0}+\Delta \theta\right)\right)^{2}=\left(0.298-0.0023 \cdot x\left(\theta_{0}+\Delta \theta\right)^{2}\right)
$$

Plugging the expression for coefficients (3) in (4) we get phase coordinates values transformation matrix from point $\Theta_{i}$ to point $\left(\Theta_{\mathrm{i}}+\Delta \Theta\right)$.

$$
\left(\begin{array}{c}
x\left(\theta_{i}+\Delta \theta\right) \\
p x\left(\theta_{i}+\Delta \theta\right)
\end{array}\right)=\left(\begin{array}{cc}
\cos \left(\omega_{i} \cdot \Delta \theta\right) & \frac{\sin \left(\omega_{i} \cdot \Delta \theta\right)}{\omega_{i}} \\
-\omega_{0} \cdot \sin \left(\omega_{i} \cdot \Delta \theta\right) & \cos \left(\omega_{i} \cdot \Delta \theta\right)
\end{array}\right) \cdot\left(\begin{array}{c}
x_{i} \\
p x_{i}
\end{array}\right)
$$

Calculating consecutively in a loop the phase coordinates values considering the changes in $\omega\left(x_{i}\left(\theta_{i}\right)\right)$ solution of the initial equation is obtained on a discrete mesh in increments $\Delta \Theta$.

Implementing any equation (1) solution algorithm there is a question of the accuracy of the solution. This equation is noncoarse (according to the accepted in nonlinear dynamics classification it means that it is particularly sensitive to computational errors and small parameter variations) and describes oscillating process in a conservative system, meaning that the amplitude is constant. Occurrence of even small errors 
leads to noncompliance of system's fundamental property (total energy is const).

In order to represent properties of the solution more thoroughly we use the methods of differential equations qualitative theory. First, we find equilibrium points, then classify them and draw a phase portrait of the system in the plane (x, px) [3].

The coordinates of equilibrium points are $a-(0,0), b-(-$ $11.4,0), \mathrm{c}-(11.4,0)$; $\mathrm{a}$ is a center, $\mathrm{b}$ and $\mathrm{c}$ are saddle points. Phase portrait appears as follows.

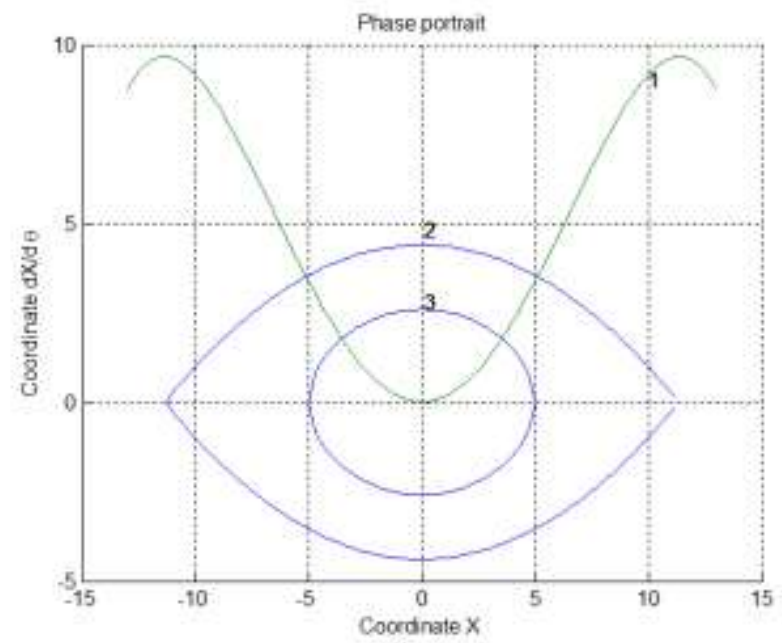

Fig.1. Phase portrait

Curve 1 is a potential function overlaying the phase portrait. Trajectory 2 is a separatrix, 3 is a trajectory of point a.

At the first stage of computational error estimation we compare qualitatively phase trajectories, computed by the proposed technique and by using ode45 [5] for the same initial conditions on the interval of argument change $\Theta=100 * \pi$ and initial values of the phase coordinates $(11,0)$.

For an exact solution phase trajectory every turn is displayed at itself. Therefore, we obtain a closed parametric curve. The graph shows that the condition fails for both algorithms, the proposed one and the numerical one.

Note that computational error of the proposed one grows faster than for the numerical one.

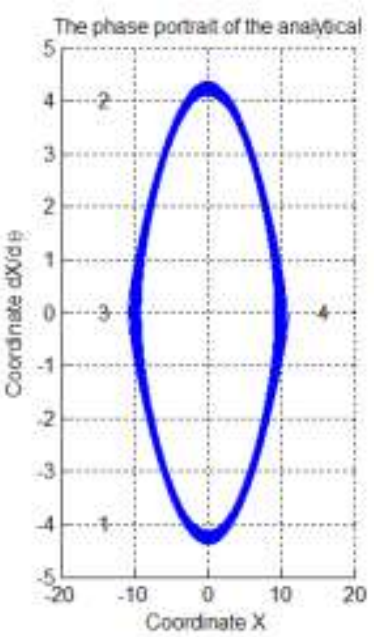

(a)

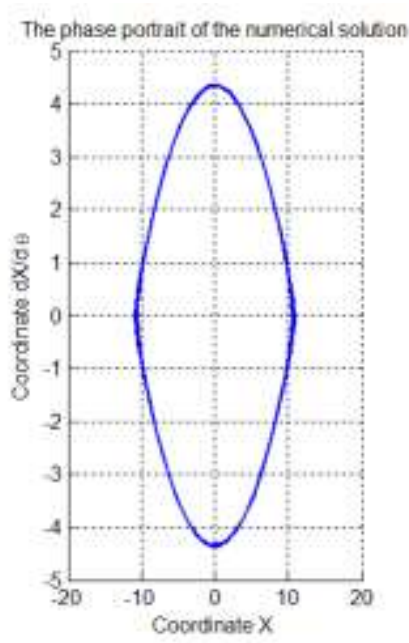

(b)
Fig 2. Phase trajectories in stable oscillations region It is especially noticeable in Fig.3a, b.

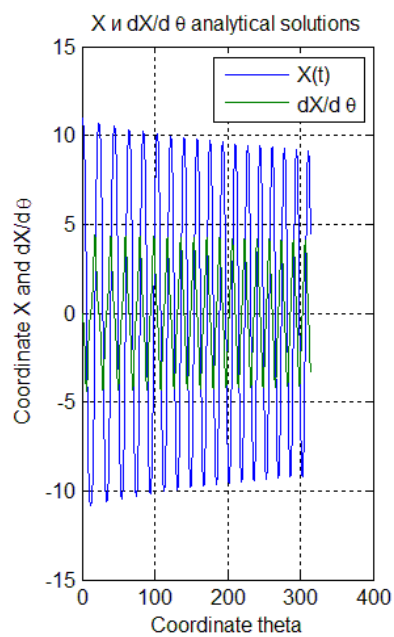

(a)

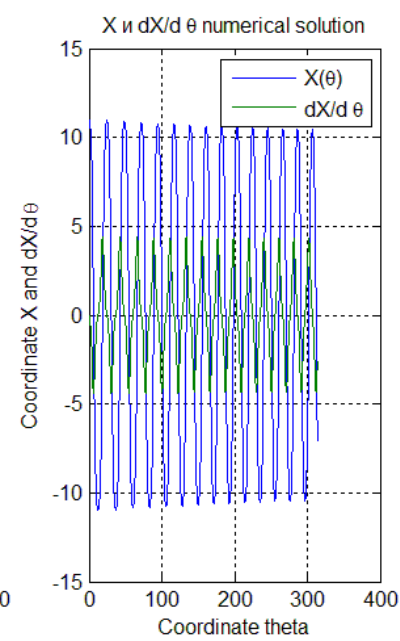

(b)
Fig. 3. Coordinates and derivatives chart.

There are two approaches to nonlinear differential equations solution in the phase plane.

1. Phase plane (x, px) moves along with the coordinate $\Theta$, so that we get continuous trajectory on the phase plane (Fig. $2 \mathrm{a}, \mathrm{b})$. This, in fact, is an extended phase space.

2. Phase plane is fixed at $\Theta_{\mathrm{s}}$ and phase coordinates values are displayed at this point, while current $\Theta$ value equals $\Theta_{\mathrm{s}}$ at $2 \pi$ intervals.

In so doing we get Poincaré map, having the number of dimensions decreased by one. According to (2a), if oscillation amplitude increases, simultaneously increases oscillation frequency and occurs phase divergence between two solutions, because speed of their amplitude decrease is different. This effect is salient at point map Fig. 4. The ability to perform this analysis of solutions, obtained by different algorithms, is 
especially important for in studies of those equations' resonating characteristic in case of periodic perturbations.

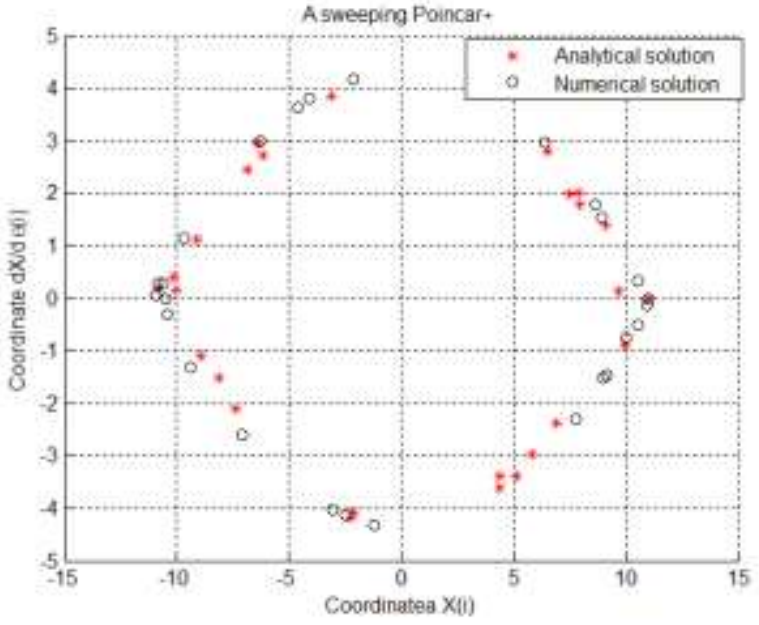

Fig. 4. Phase trajectories Poincaré point map

\section{EXACT SOLUTION}

The proposed algorithm can obtain exact solution of the equation (1) if the total energy loss is compensated at each step. Kinetic energy as a function of speed is calculated using well-known formula. Potential energy is an integral of restoring force in (1)

$$
\Pi=\int\left(0.298 \cdot x-0.0023 \cdot x^{3}\right) d x=0.149 \cdot x^{2}-0.000575 \cdot x^{4} .
$$

Total energy can be found as a sum of kinetic and potential energy. Plug the initial conditions $\left(\mathrm{x}_{0}, \mathrm{px}_{0}\right)$ in expression for total energy to find $\mathrm{W}_{0}$

$$
W_{0}=p x_{0}^{2} / 2+0.149 \cdot x_{0}^{2}-0.000575 \cdot x_{0}{ }^{4} .
$$

Amplitude is decreasing follows the law that is close to exponential. So the expression for the total energy can be written as

$$
W_{i}=e^{k \cdot \Delta} \theta \cdot W_{0} .
$$

Correction factor can be found from (6). With regard to squares of coordinates and derivatives in (5), exact values of phase coordinates are

$$
\begin{gathered}
x_{t i}=\sqrt{e^{-\ln \left(W_{i} / W_{0}\right)}} \cdot x_{i} \\
p x_{t i}=\sqrt{e^{-\ln \left(W_{i} / W_{0}\right)}} \cdot p x_{i} .
\end{gathered}
$$

Corrected (7) solution (1) is displayed at Fig. 5. Initial conditions and integration interval are identical to the first phase portrait Fig. 2.

The figure shows that the points at analytical solution phase trajectory are displayed at itself. It means that total energy is const.

This is also confirmed by Fig. 6a, where oscillation amplitude remains const.

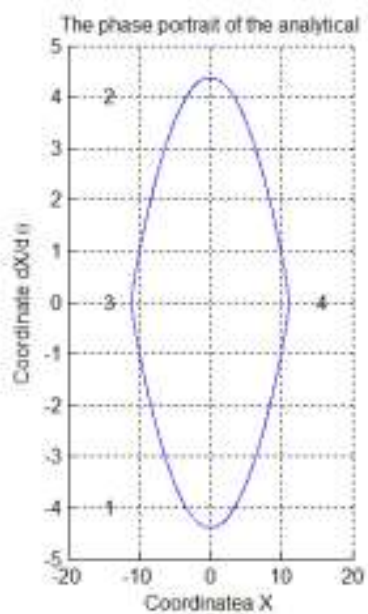

(a)

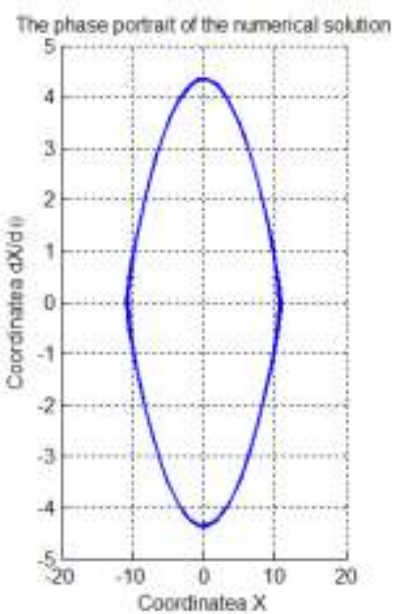

(b)
Fig. 5. Exact analytical solution

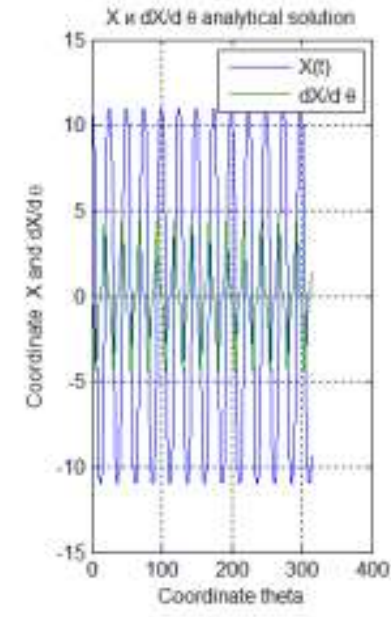

(a)

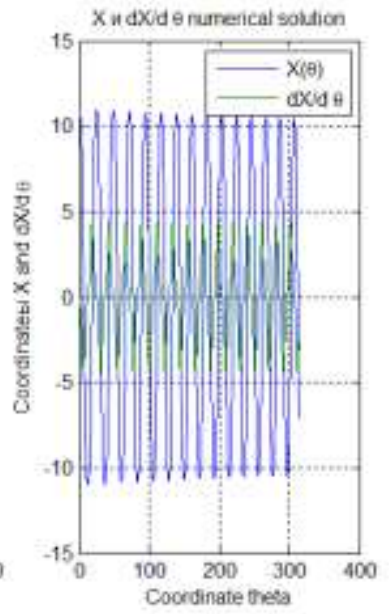

(b)
Fig. 6. Coordinates and derivatives plot

Consider estimation of the solution using Poincaré secant. 


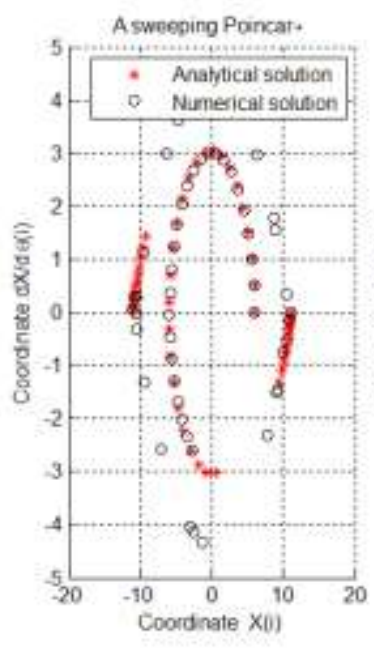

(a)

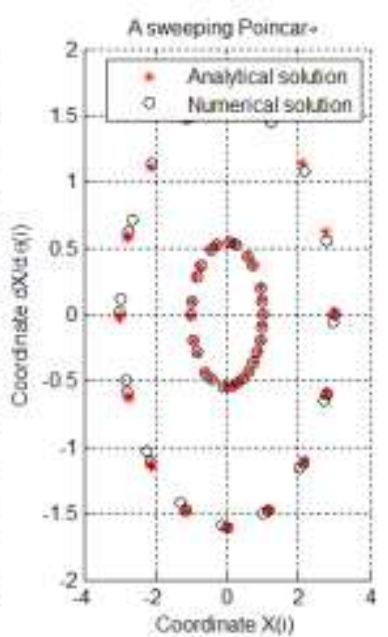

(b)
Fig. 7. Poincaré secant for exact solution

There is a Poincaré map for different initial conditions at Fig. 7b. For $\left(\mathrm{x}_{0}=1, \mathrm{px}_{0}=0\right)$ frequency according to (2) changes slowly and numerical solution approximately coincide with analytical solution. In case $\left(\mathrm{x}_{0}=3, \mathrm{px}_{0}=0\right)$ the first signs of phase divergence occur, which becomes more and more noticeable as oscillation amplitude (initial conditions) increases Fig. 7a.

In order to perform more objective analysis for both solutions use property of differential equation solution. Plugging the solution in differential equation reduces it to an identity. For a homogenous equation, there must be zero as a result.

According to the results, shown in Fig. 7 it is seen that analytical solution error is two degrees less then numerical solution error. In addition, Fig. 6 shows that numerical method came out with three more oscillation than analytical one since the frequency increases as oscillation amplitude according to (2a) decreases.

Furthermore, it should be noticed that for plotting analytical solution phase portrait Fig. 2 the number of steps is larger then number of steps for numerical solution. Corrected according to (7) analytical method needs twice less steps then the numerical one.

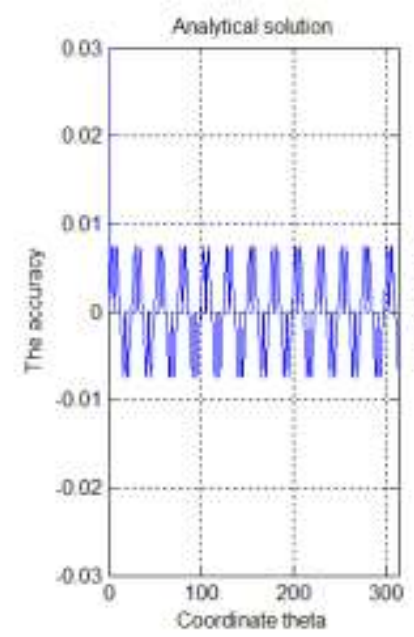

(a)

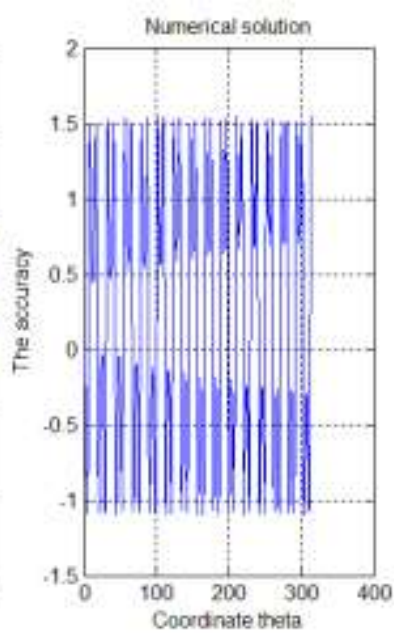

(b)
Fig. 8. Computational error of (a) analytical and (b) numerical solution.

\section{SOLUTION IN UNSTABLE OSCILLATIONS REGION}

If the initial conditions are defined in a way that that mapping point at the phase plane lies outside separatrix, then according to (2a) $\omega(\mathrm{x})^{2}<0$.

Equation (2) is written as

$$
\frac{d^{2} x}{d \theta^{2}}-k(x)^{2} \cdot x=0 .
$$

In this case fundamental system of solution functions are

$$
\exp (-k \cdot \theta) ; \exp (k \cdot \theta)
$$

general solution and it's derivative are written as

$$
\begin{aligned}
& x(\theta)=a \cdot \exp (-k \cdot \theta)+b \cdot \exp (k \cdot \theta), \\
& \frac{d x}{d \theta}=-k \cdot a \cdot \exp (-k \cdot \theta)+k \cdot b \cdot \exp (k \cdot \theta) .
\end{aligned}
$$

Method of equation (7) solution is the same as method for (1). The only distinction is that trigonometric functions are replaced with exponents.

Equation (7) unlike the previous case is coarse. Properties of coarse equations are not changing a lot if parameters are changed slightly or there is a negligible error. Suggested and numerical solutions are compared without refining.

Phase portrait is plotted at Fig. 9. There are shown trajectories for four different initial conditions. Qualitatively, phase trajectories built with both methods are almost the same. 


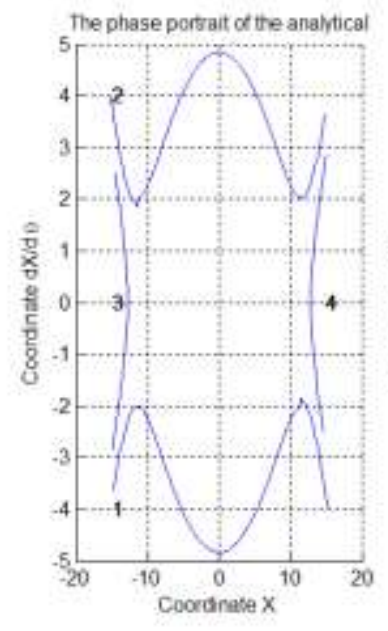

(a)

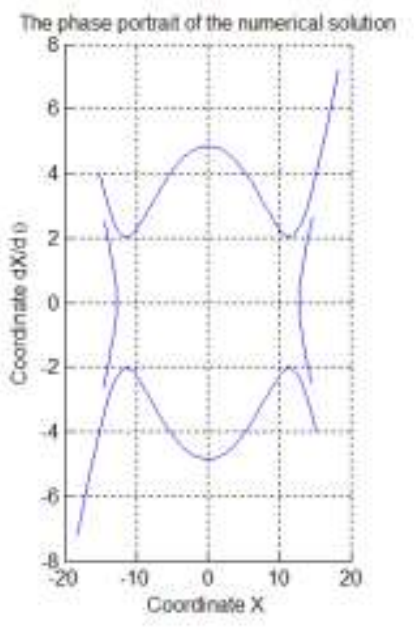

(b)
Fig. 9. Phase trajectories in unstable oscillations region

Having plugged the solutions in equation (7) one can see that analytical solution error is a degree less then numerical solution error. That provides support to the fact the analytical solution is close to explicit solution (Fig. 10).

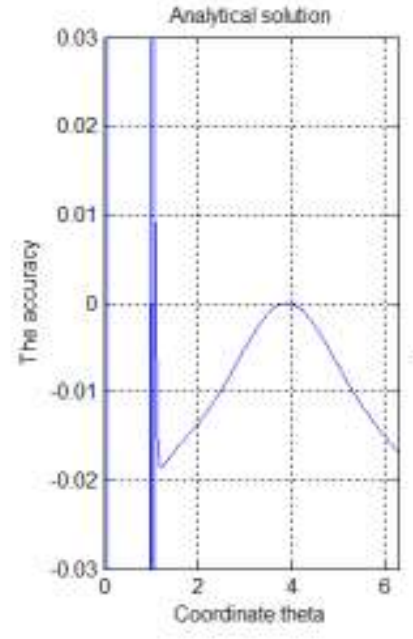

(a)

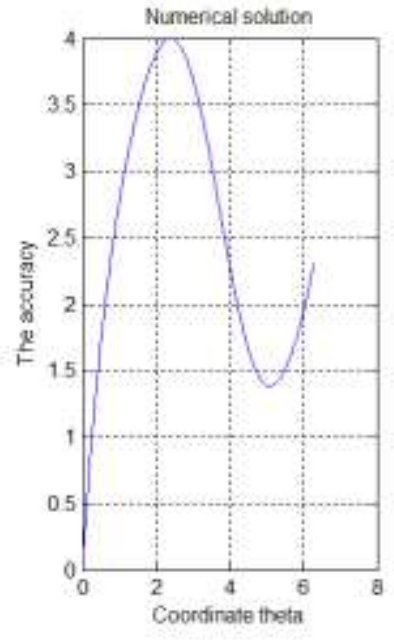

(b)
Fig. 10. Computational error of (a) analytical and (b) numerical solution.

On the base of conducted research, one can make a conclusion:

1. Design and using of algorithms that take into account features of mathematical models of specific physical systems (in particular, total energy conservation law) let us obtain more accurate solution then built-in integration functions of mathematical software packages.

2. In recent years analytical mathematical packages such as Mathematica, Maple are used more and more frequently for studying theory of differential equations and for analytical solution of specific tasks [4]. Suggested solution method lets enhance the abilities of those packages, because it can be implemented with built-in functions of the packages.

\section{REFERENCES}

[1] L. M. Ananiev, A. A. Vorobiev, B. I. Gorbunov, "Induction electron accelerator, betatron" Moscow, pp. 350, 1961.

[2] N. M. Matveev, "Integration of ordinary differenrial equations" Visschaya Schkola, Moscow, 2005.

[3] N. N. Bautin, E. A. Leontovich, "Methods and techniques of qualitative research of dynamical system in a plane", Nauka. Moscow, 1990.

[4] C. H. Edwards, D. E. Penny, "Differential equations and boundary value problems: computing and modeling" Moscow, 2008.

[5] I. E. Anufriev, A. B. Smirnov, E. N. Smirnova "MATLAB in the original" SPb: BHV, St. Peterburg, 2005. 
